\title{
Übernahmeaufforderungen beim hochautomatisierten Fahren mit fahrfremden Tätigkeiten - welche Modalitäten sind geeignet?
}

\author{
Andreas Lars Müller ${ }^{1}$ Bettina Abendroth ${ }^{1}$ \\ Angenommen: 12. November 2021 / Online publiziert: 9. Dezember 2021 \\ (c) Der/die Autor(en) 2021
}

\section{Zusammenfassung}

Hochautomatisierte Fahrzeuge werden in der Lage sein, die Fahrzeugführung selbständig zu übernehmen, während sich die Fahrer_innen mit fahrfremden Tätigkeiten beschäftigen können. In kritischen Situationen, die das Automatisierungssystem nicht allein bewältigen kann, wird es jedoch weiterhin Übernahmeaufforderungen an den Fahrer/die Fahrerin geben. In diesem Beitrag wird untersucht, welche Modalitäten für Übernahmeaufforderungen entsprechend einer Reiz-ReaktionsKompatibilität geeignet sind. Dazu wurden drei unterschiedliche Schnittstellen-Varianten entwickelt und mit insgesamt 126 Proband_innen in zwei unabhängigen Fahrsimulator-Studien überprüft. Innerhalb der ersten Studie wurden eine visuelle, eine vibrotaktile oder eine multimodale (Kombination aus visueller, vibrotaktiler und akustischer) Übernahmeaufforderung untersucht. Die zweite Studie konzentrierte sich auf die mentale Beanspruchung und die Rückübernahmefähigkeit bei Ausführung verschiedener fahrfremder Tätigkeiten während hochautomatisierter Fahrt. Diese Studie ergab, dass die Dauer der Kontrollübernahme nach einer multimodalen Rückübernahmeaufforderung mit der mentalen Beanspruchung der fahrfremden Tätigkeiten korreliert.

Praktische Relevanz: Die Untersuchung unterschiedlicher Modalitäten für die Informationsübermittlung bei einer Rückübernahmeaufforderung trägt dazu bei, dass in automatisierten Fahrzeugen Modalitäten gewählt werden, die zum einen eine kurze Reaktionszeit der Fahrer_innen unterstützen und zum anderen eine subjektiv positive Bewertung hervorrufen.

Schlüsselwörter Automatisiertes Fahren · Rückübernahmeaufforderung · Reaktionszeit · Fahrfremde Tätigkeiten · Mentale Beanspruchung

\section{Takeover requests at conditional automated driving with non-driving related activities-which are suitable modalities?}

\begin{abstract}
Conditional automated vehicles will be able to take over the driving of the vehicle independently, while the drivers can occupy themselves with non-driving activities. However, in critical situations that the automation system cannot handle on its own, there will still be takeover requests to the driver. This paper investigates which modalities are suitable for takeover requests according to stimulus-response compatibility. For this purpose, three different interface variants were developed and tested with a total of 126 test subjects in two independent driving simulator studies. Within the first study, a visual, a vibrotactile or a multimodal (combination of visual, vibrotactile and acoustic) takeover request was investigated. The second study focused on the mental demands and the ability to take over when performing different non-driving tasks during highly automated driving. This study found that the duration of control takeover after a multimodal takeover request correlated with the mental demands of the non-driving activities.

Practical Relevance: The investigation of different modalities for the transmission of information in take-over-situations contributes to the implementation of modalities in automated vehicles that on the one hand support a short reaction time of the driver and on the other hand cause a subjectively positive evaluation.
\end{abstract}

Dr.-Ing. Bettina Abendroth

bettina.abendroth@tu-darmstadt.de
Fachbereich Maschinenbau, Institut für Arbeitswissenschaft, Technische Universität Darmstadt, Otto-Berndt-Straße 2, 64287 Darmstadt, Deutschland 
Keywords Automated driving $\cdot$ Take-over request $\cdot$ Reaction time $\cdot$ Non-driving related activities $\cdot$ Mental workload

\section{Fragestellung}

Automatisiertes Fahren ist derzeit einer der treibenden Faktoren in der Automobilbranche. Die technische Entwicklung schreitet voran und erste Automatisierungssysteme sind bereits verfügbar. Es wird jedoch Situationen geben, in denen solche Systeme an ihre Grenzen stoßen. In diesen Fällen muss der Fahrer/die Fahrerin eingreifen und die Kontrolle über das Fahrzeug zeitnah und mit einer hohen Übernahmequalität übernehmen.

Dieser Beitrag bezieht sich auf die Automatisierungsstufen der SAE J3016 (2021). Neben dem manuellen Fahren (Level 0) gibt es seit der Einführung des adaptiven Tempomaten im Jahr 1998 das assistierte Fahren (Level 1). Bei der Teilautomatisierung (Level 2) übernimmt das Fahrzeug selbstständig die Stabilisierung und der Fahrer überwacht das System auf der Bahnführungsebene (Donges 1982). Beim hochautomatisierten Fahren (Level 3) wird davon ausgegangen, dass sich der Fahrer/die Fahrerin für eine gewisse Zeit vom aktiven Fahren abwenden und sich fahrfremden Tätigkeiten (FFT) widmen kann. Nach der Definition der SAE J3016 (2021) und der NHTSA (2012) sind Fahrer_innen bei der hochautomatisierten Fahrt weiterhin verpflichtet, die Fahrzeugkontrolle innerhalb einer bestimmten Zeitspanne zu übernehmen, wenn sie durch eine Übernahmeaufforderung (TOR, Take-Over-Request) dazu aufgefordert werden. In diesem Fall fungiert der Mensch als Rückfallebene für das Automatisierungssystem.

Damit ein TOR eine zeitlich angemessene Rückübernahme bewirkt, ist neben der Wahrnehmbarkeit die ReizReaktions-Kompatibilität bei der Gestaltung des MenschMaschine-Systems zu gewährleisten, um den Umkodieraufwand in der Informationsverarbeitung zu reduzieren und mentale Kapazitäten zu entlasten (Strasser 2008).

Dementsprechend wurden in einer ersten Studie unterschiedliche Modalitäten des TOR hinsichtlich ihrer Wahrnehmbarkeit basierend auf folgender Forschungsfrage 1 untersucht: Ist ein unimodaler TOR ausreichend, um eine schnelle Reaktion des Fahrers/der Fahrerin zu erzielen oder führt eine gleichzeitige multimodale Ansprache verschiedener Sinneskanäle zu besseren Reaktionszeiten? Darüber hinaus soll untersucht werden, ob es eine direkte Handlungsaufforderung nach verschiedenen TOR-Modalitäten gibt und wie störend insbesondere der visuelle und akustische TOR wahrgenommen wird.

Die Auswirkungen von fahrfremden Tätigkeiten auf die mentale Beanspruchung und die Rückübernahmefähigkeit wurden in einer zweiten Studie analysiert. Da sich die Fahrzeugnutzenden bei hochautomatisierter Fahrt vom Fahrgeschehen abwenden und sich FFT zuwenden können, stellt sich die Frage, ob die Art der FFT einen Einfluss auf die Rückübernahmezeit nach einem TOR hat. Wie sich unterschiedliche FFT auf die Übernahmefähigkeit auswirken, soll mit folgender Forschungsfrage 2 genauer untersucht werden: Wie unterscheidet sich die Rückübernahmefähigkeit bei der Ausführung verschiedener FFT?

\section{Theoretische Grundlagen}

Frühere Studien befassten sich bereits mit einigen Faktoren, die die Rückübernahme von Fahrer_innen beim automatisierten Fahren beeinflussen. Radlmayr et al. (2014) wiesen bereits nach, dass die Verkehrsdichte beim Fahren auf der Autobahn einen signifikanten Einfluss hat. Darüber hinaus kamen die Autoren zu dem Schluss, dass FFT, wie beispielsweise die Nutzung eines Smartphones, die Übernahmequalität in Situationen mit dichtem Verkehr verschlechtert und die Wahrscheinlichkeit von Kollisionen erhöht. Eriksson et al. (2015) fanden bei einer Online-Befragung mit Bildern verschiedener komplexer Verkehrssituationen heraus, dass die Orientierung in weniger komplexen Situationen und unter Zeitdruck schneller erfolgte. Merat et al. (2012) haben festgestellt, dass die Reaktionen auf kritische Vorfälle beim automatisierten Fahren ohne FFT den Reaktionen in Situationen beim manuellen Fahren ähneln. Carsten et al. (2012) untersuchten in einer Fahrsimulatorstudie die Auswirkungen von drei verschiedenen Automatisierungsleveln (manuell, teilautomatisiert und hochautomatisiert) auf die Aufmerksamkeit von Fahrer_innen auf den Straßenverkehr bei gleichzeitiger Ausführung von FFT. Die Ergebnisse zeigen, dass die Beschäftigung mit FFT mit einem höheren Automatisierungsgrad zunimmt, was gleichzeitig zu einer Abnahme der Fokussierung des Fahrers/der Fahrerin auf den Straßenverkehr führt. Strand et al. (2014) bestätigten den negativen Einfluss von hohen Automatisierungsgraden auf die Fahrleistung nach einer Rückübernahme beim Vergleich zwischen teil- und hochautomatisierter Fahrzeugführung in kritischen Situationen. Happee et al. (2017) führten eine Fahrsimulatorstudie durch, deren Ziel es war, ein Überholmanöver auf einer Autobahn mit einer blockierten Fahrspur zu untersuchen. Im Rahmen ihrer Untersuchung konnten sie einen negativen Einfluss höherer Automatisierungsgrade auf die Rückübernahme nachweisen, da die Lenk- und Bremseingriffe bei automatisierter Fahrt im Vergleich zum manuellen Fahren verzögert erfolgten. Damböck et al. (2012) untersuchten die Zeit, die für einen Wechsel vom autonomen Fahren zurück zum manuellen Fahren benötigt wird, um einen für den Fahrer komfortablen Übernahmeprozess zu ermöglichen. Auf der Grundla- 
ge ihrer Ergebnisse schlagen die Autoren einen Zeitrahmen von mindestens sechs Sekunden vor, der für einen komfortablen Wechsel erforderlich ist. Andere Studien konzentrieren sich auf die Erforschung verschiedener Sinneskanäle bei einem TOR.

Naujoks et al. (2014) untersuchten die Auswirkungen von visuell-auditiven im Vergleich zu rein visuellen TORs auf den Fahrer/die Fahrerin bei automatisierter Fahrt. Sie haben festgestellt, dass die Reaktionszeit „Hände am Lenkrad" nach einem visuell-auditiven TOR signifikant kürzer war. Petermeijer et al. (2017a) zeigten positive Funktionen eines vibrotaktilen Feedbacks im Vergleich zu einem auditiven TOR und einer Kombination aus beiden. Die Experimente wurden auf einer simulierten dreispurigen, geraden Autobahn ohne Verkehr und einer Fahrgeschwindigkeit von $120 \mathrm{~km} / \mathrm{h}$ durchgeführt. Im Rahmen ihrer Studie wurden die Reaktionszeiten der Fahrer_innen während der Ausführung von FFT bewertet. Die Ergebnisse zeigen, dass Lenkeingriffe bei visuell-auditiven TORs am schnellsten ausgeführt werden können. Die Richtung des Ausweichens nach einem TOR ist unabhängig davon, ob der Warnton und/oder die Vibration links oder rechts ausgegeben werden. Petermeijer et al. (2017b) untersuchten die Wirkung verschiedener Varianten eines vibrotaktilen TOR in einer Fahrsimulatorstudie. Die Ergebnisse zeigten, dass die Teilnehmer schneller reagierten, wenn die Vibration über die gesamte Sitzfläche wahrnehmbar war, im Vergleich zu einzelnen Vibrationsmustern ausgeführt wurden. Telpaz et al. (2017) führten Experimente mit vibrotaktilem Feedback durch, nachdem die Teilnehmenden aufgefordert wurden, während des autonomen Fahrens eine Textnachricht von einem Smartphone zu senden. Mittels einer Vibration wurde ein Hinweis auf den Verkehr wiedergegeben. Die Studie fand auf einer simulierten fünfspurigen Autobahn statt. Die Autoren stellten fest, dass die Reaktionszeiten bei einem vibrotaktilen TOR schneller als bei einem rein akustischen TOR waren.

Zur Untersuchung von FFT im Fahrkontext werden in der Literatur standardisierte und naturalistischere FFT un- terschieden. Standardisierte Techniken, welche naturalistischere FFT imitieren sollen, sind beispielsweise der n-Back Task (Kirchner 1958) oder die visuelle Suchaufgabe SuRT nach DIN ISO/TS 14198 (2019). Als Nachteil standardisierter Tests kann die fehlende Übertragbarkeit der Ergebnisse auf die Realität angesehen werden. Ebenso ist die Motivation der Aufgabenausführung bei naturalistischeren FFT vermeintlich höher als bei standardisierten Tätigkeiten, was die Übernahmezeit verlängern kann.

Petermann-Stock et al. (2013) untersuchten unterschiedlich belastende FFT während hochautomatisiertem Fahren. Mehrere Versionen eines Quizspiels wurden dafür in ein Experiment implementiert, um eine steigende Arbeitsbelastung zu simulieren. Die größte Übernahmefähigkeitsbeeinträchtigung wurde für die Variante festgestellt, die eine Kombination aus akustischer, kognitiver, visueller und motorischer Belastung beinhaltete. In einer Studie von Vogelpohl et al. (2016) führten die Teilnehmenden auf einem Tablet zwei FFT aus (Lesen eines Zeitungsartikels, TetrisSpiel) und verglichen beide FFT mit einer Referenzfahrt. Im Vergleich zu einer Kontrollgruppe ohne FFT waren die Übernahmezeiten für beide FFT signifikant länger. Der Vergleich zwischen den FFT ergab keinen signifikanten Unterschied. In Gold und Körber et al. (2016) und in Louw et al. (2015) fanden sich im Gegensatz zu den eben genannten Ergebnissen keine signifikanten Unterschiede zwischen abgelenkten und nicht abgelenkten Fahrenden. Zeeb et al. (2016) untersuchten ebenfalls den Einfluss naturalistischer FFT (E-Mail schreiben, News lesen und Video schauen) auf die Übernahmeleistung. Daraus ergaben sich keine signifikanten Auswirkungen auf die Reaktionszeiten (Hand ans Lenkrad) innerhalb der untersuchten FFT.

\section{Methodik}

Zur Untersuchung von Forschungsfrage 1 wurden drei verschiedene TORs entwickelt und in drei unabhängigen Stu-

Tab. 1 Übersicht der durchgeführten Studien zur Evaluation der TOR Gestaltung

Table 1 Overview of the studies conducted to evaluate the TOR design

\begin{tabular}{|c|c|c|c|c|c|}
\hline Studie & TOR & $\begin{array}{l}\text { Untersuchungs- } \\
\text { umgebung }\end{array}$ & FFT & Probanden & Untersuchungsziel \\
\hline $1 a$ & Vibrotaktil: Vibrationsmatte & $\begin{array}{l}\text { Fahrzeug Mock- } \\
\text { up, keine Fahrsi- } \\
\text { mulation }\end{array}$ & - & $N=21$ & Wahrnehmung der Vibrationen \\
\hline $1 b$ & Visuell: LED-Lichtband & $\begin{array}{l}\text { Fahrzeug Mock- } \\
\text { up, mit Fahrsi- } \\
\text { mulation }\end{array}$ & Smartphone & $N=19$ & $\begin{array}{l}\text { Reaktionszeit, subjektive Be- } \\
\text { wertung }\end{array}$ \\
\hline $1 c$ & $\begin{array}{l}\text { Vibrotaktil: Vibrationsmatte } \\
\text { Multimodal: Vibrationsmatte, } \\
\text { LED Lichtband, Warnton }\end{array}$ & $\begin{array}{l}\text { Fahrzeug Mock- } \\
\text { up, mit Fahrsi- } \\
\text { mulation }\end{array}$ & 2-Back-Test (Tablet) & $N=30$ & $\begin{array}{l}\text { Reaktionszeit, subjektive Be- } \\
\text { wertung }\end{array}$ \\
\hline 2 & $\begin{array}{l}\text { Multimodal: Vibrationsmatte, } \\
\text { LED Lichtband, Warnton }\end{array}$ & $\begin{array}{l}\text { Fahrzeug Mock- } \\
\text { up, mit Fahrsi- } \\
\text { mulation }\end{array}$ & $\begin{array}{l}\text { Text lesen, Hörbuch hören, } \\
\text { Video schauen, Texting, Stra- } \\
\text { ße beobachten (Referenz) }\end{array}$ & $N=56$ & $\begin{array}{l}\text { Reaktionszeit in Abhängigkeit } \\
\text { der unterschiedlich mental } \\
\text { beanspruchenden FFT }\end{array}$ \\
\hline
\end{tabular}


dien mit Proband_innen im Fahrsimulator des Instituts für Arbeitswissenschaft an der TU Darmstadt (IAD) evaluiert, siehe Tab. 1.

In Studie $1 a$ wurde die wahrgenommene Vibrationsintensität untersucht. Ziel war es, die optimale Vibrationsstärke eines vibrotaktilen TOR zu ermitteln.

In Studie $1 b$ wurde ein visueller TOR getestet. Die Proband_innen haben ihr Smartphone während der automatisierten Fahrt als FFT verwendet.

Der vibrotaktile TOR und der multimodale (visuelle, vibrotaktile und akustische) TOR wurden in Studie $1 c$ auf ihre Reaktionszeit hin untersucht. Als FFT haben die Proband_innen einen standardisierten 2-Back-Test durchgeführt.

Durch die FFT wurden die Proband_innen so weit wie möglich vom Fahrgeschehen abgelenkt und somit vergleichbare Untersuchungsbedingungen geschaffen. Zusätzlich zu den objektiven Fahrdaten aus dem Simulator wurden in allen Studien subjektive Daten mittels Fragebögen erhoben. Die Daten aus Studie $l b$ und Studie $1 c$ werden miteinander verglichen und daraus eine Gestaltungsempfehlung für einen optimierten TOR abgeleitet.

Zur Untersuchung der zweiten Forschungsfrage wurden vier FFT (Text lesen, Hörbuch hören, Video schauen und Texting) sowie eine Referenztätigkeit (Straße beobachten) während einer hochautomatisierten Fahrt in einem simulierten Stadtkurs ausgeführt. Die mentale Beanspruchung während der Ausführung der einzelnen FFT wurde durch eine Sekundäraufgabe in Form eines Detection Response Tasks nach dem Subsidiary-Task-Paradigm analysiert.

\subsection{Untersuchte TOR-Varianten}

Durch ein TOR wird der Fahrer/die Fahrerin darüber informiert, dass sie die Fahrzeugführung vom Automatisierungssystem zurückübernehmen sollen. Ein TOR können den Fahrer_innen über verschiedene Modalitäten mitgeteilt werden. Auf Grundlage der Literaturerkenntnisse wurden die untersuchten TORs auf die visuelle, vibrotaktile und akustische Modalität beschränkt.

\subsubsection{Visueller TOR}

Untersuchungen haben gezeigt, dass LED-Lichtleisten im Vergleich zu klassischen visuellen Anzeigen ein großes Potenzial als visuelles Warnsignal haben und, dass weniger Blickabwendungen von der Straße auftreten, da Warnungen auch im peripheren Sichtfeld wahrgenommen werden (Utesch 2014). Die LED-Anordnung um den Fahrer/die Fahrerin kann auch für räumlich orientierte Warnungen genutzt werden.

Für das visuelle Informations- und Warnsystem wurden drei LED-Lichtleisten (LPD8806) am Fahrzeug-Mock-up

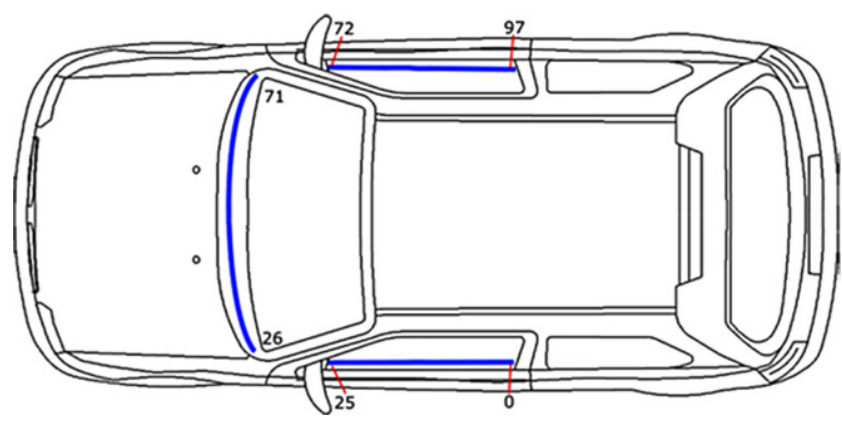

Abb. 1 Positionierung der LED Lichtbänder am Fahrzeug-Mock-up

Fig. 1 Positioning of the LED light strips on the vehicle mock-up

des Fahrsimulators angebracht, die insgesamt 97 einzeln ansteuerbare LEDs umfassen. Je eine LED-Leiste wurde im Sichtfeld des Fahrers/der Fahrerin an der Fahrer- und Beifahrertür und eine dritte auf dem Armaturenbrett in Höhe der Windschutzscheibe positioniert, siehe Abb. 1. Bei der Entwicklung und Konstruktion wurde darauf geachtet, dass die LED-Lichtleisten auch bei der Ausführung von FFT und somit beim Abwenden vom aktuellen Verkehrsgeschehen sichtbar sind. Die LED Leisten strecken sich im horizontalen Bereich auf ca. 180-200 aus und decken somit das gesamte Blickfeld des Menschen ab. Mit Hilfe eines Arduino-Mikrocontrollers wurden die LED-Lichtleisten hinsichtlich ihrer Helligkeit, Farbe und Blinkfrequenz dynamisch gesteuert und mit der Simulationssoftware verbunden. Während eines visuellen TOR leuchteten die LEDs in Rot und pulsierten mit einer Frequenz von $2,6 \mathrm{~Hz}$.

\subsubsection{Akustischer TOR}

Akustische Signale werden unabhängig von der Blickrichtung des Fahrers/der Fahrerin wahrgenommen und spielen daher eine wichtige Rolle bei der Durchführung einer FFT. Zudem sind sie omnidirektional und können aus jeder Richtung wahrgenommen werden. In Anlehnung an die Theorie der multiplen Ressourcen von Wickens (2008) lassen sich weitere Vorteile erkennen, da eine parallele Verarbeitung von akustischen Signalen und visuellen Informationen möglich ist. Hinsichtlich der Auswahl eines geeigneten Signaltons wurde eine Studie von Färber (1987) als Referenz herangezogen. In dieser Studie mussten die Proband_innen verschiedene Tonfrequenzen in Bezug auf Dringlichkeit und Annehmlichkeit bewerten. Auf der Grundlage der Ergebnisse wurde ein $75 \mathrm{~dB}(\mathrm{~A})$ Sinuston mit $440 \mathrm{~Hz}$ und einer Dauer von einer Sekunde, der alle zwei Sekunden ertönt, als akustischer TOR ausgewählt.

\subsubsection{Vibrotaktiler TOR}

Der Informationsgehalt von vibrotaktilen Signalen ist im Vergleich zu visuellen oder akustischen Signalen begrenzt. 
Vibrotaktile Informationen sind jedoch unabhängig von Sichtfeld und Umgebungsgeräuschen für die fahrende Person wahrnehmbar, ohne dass diese weitere Passagiere im Fahrzeug stören. Die Sicherheitsgurte und die Sitze sind die einzigen Flächen im Fahrzeug, mit denen Fahrer_innen immer physisch in Kontakt sind, so dass diese für die Umsetzung vibrotaktiler Informationen gut geeignet sind (Petermeijer et al. 2017a). Daher wurde für diese Untersuchung eine Vibrationsmatte konstruiert, die sowohl im IAD-Fahrsimulator als auch in realen Fahrzeugen verwendet werden kann. Bei der Auslegung der Vibrationssignale wurden die Ergebnisse von Ji et al. (2011) berücksichtigt. Diese führten verschiedene Studien zum Intensitätsbereich der vibrotaktilen Aktuatoren sowie zur räumlichen Anordnung von Aktuatoren durch.

Die verwendeten Schwingungsaktuatoren weisen eine ähnliche Charakteristik auf wie die von Ji et al. (2011) vorgeschlagenen. Es wurde eine Vibrationsmatte mit 21 exzentrischen Massenrotationsaktuatoren (Precision Microdrives 320-105) in einer 7×3-Anordnung entwickelt, siehe Abb. 2.

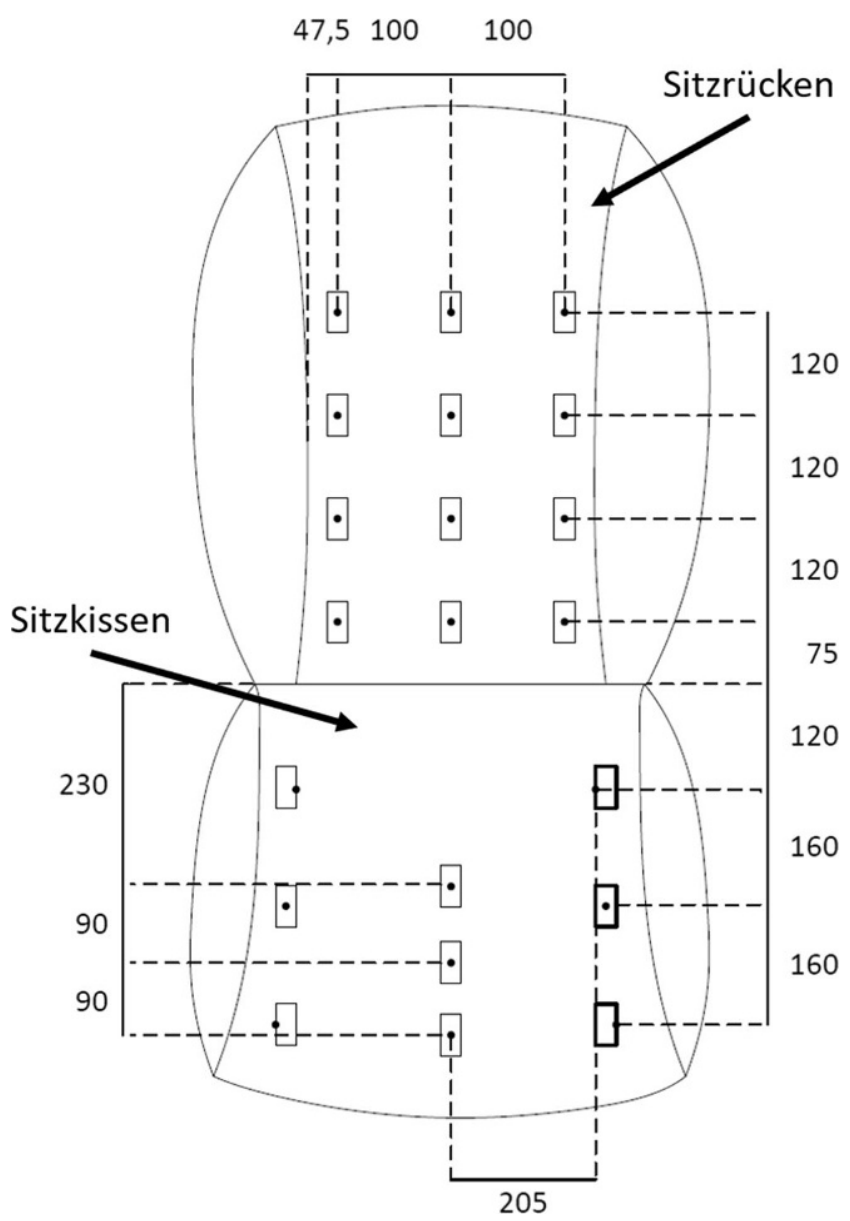

Abb. 2 Anordnung der Vibrationsaktuatoren mit $21(7 \times 3)$ Unwuchtmotoren; alle Abstände sind in Millimetern angegeben

Fig. 2 Vibrotactile mat arrangement layout with $21(7 \times 3)$ vibration motors; all distances are given in millimeters
Die Matte ist in der Lage, sowohl dynamische als auch statische Schwingungsmuster zu übertragen und kann auf dem Fahrersitz positioniert werden. Jeder der Aktuatoren kann separat angesteuert werden, dies erfolgt über einen Arduino-Mikrocontroller mit einer selbst entwickelten Software, um die Verbindung mit der Simulationssoftware zu gewährleisten.

Die tragbare vibrotaktile Matte besteht aus $2,5 \mathrm{~cm}$ dickem Schaumstoff. Für die Aktuatoren sind zwölf Aussparungen in der Rückenlehne und neun Aussparungen im Sitzkissen vorgesehen. Die Aktuatoren befinden sich in schützenden Kunststoffrohren, die in die Aussparungen eingesetzt wurden. Der Schaumstoff weist einen hohen Härtegrad auf, um ein Einsinken der Benutzer_innen zu verhindern und ein bequemes Sitzen zu ermöglichen.

\subsection{Untersuchungskonzept}

Die Versuche wurden in dem statischen Fahrsimulator am Institut für Arbeitswissenschaft der Technischen Universität Darmstadt durchgeführt. Der Fahrsimulator besteht aus einem vollständigen Fahrzeug-Mock-up (Chevrolet Aveo), einem Sichtfeld von $180^{\circ}$ Frontprojektion und einer Darstellung aller Fahrspiegel durch drei Rückprojektionen. Die Simulation wird mit Silab 5.1 (WIVW) und einem selbstentwickelten Automatisierungscontroller auf Basis der Definition von SAE J3016 (2021) Level 3 hochautomatisiertes realisiert.

In Studie $1 a$ wurde nur die vibrotaktile Vibrationsmatte ohne simulierte Fahraufgabe untersucht. In Studie $1 b$ und Studie $1 c$ wurden insgesamt drei verschiedene ÜbernahmeSzenarien eingesetzt, die aufgrund des Ausfalls des Automatisierungssystems notwendig waren (siehe Tab. 1).

Es wurde ein $3 \times 3$-Versuchsplan unter Berücksichtigung der drei Übernahmeszenarien sowie der drei TOR-Varianten visuell, vibrotaktil und ein multimodal (Kombination aus visuellem, vibrotaktilem und akustischem TOR) erstellt.

Zwischen dem vibrotaktilen und dem multimodalen TOR sowie zwischen dem visuellen und dem vibrotaktilen TOR wurde ein Within-Subject-Design gewählt, während ein Between-Subject-Design für den Vergleich der Varianten visueller und multimodaler TOR zum Einsatz kam. In allen Szenarien mussten die Proband_innen nach einem TOR die FFT unterbrechen, um die Fahrzeugführung $\mathrm{zu}$ übernehmen. $20 \mathrm{~s}$ nach der erfolgreichen Übernahme pausierte die Simulation für die Beantwortung eines Fragebogens. Danach folgte das nächste Szenario. Insgesamt dauerte das Fahrsimulatorexperiment $30 \mathrm{~min}$ in Studie $1 b$ und $60 \mathrm{~min}$ in Studie $1 c$.

In Studie $1 a$ wurde ein Fragebogen zur Bewertung der wahrgenommenen Vibrationen auf einer 7-stufigen LikertSkala (sehr angenehm-sehr unangenehm) eingesetzt. Die Proband_innen haben zwölf verschiedene Vibrationsstärken 
bewertet, die in permutierter Reihenfolge für jeweils $5 \mathrm{~s}$ nacheinander dargeboten wurden.

Um die verschiedenen TOR-Varianten in Studie $1 b$ und Studie $1 c$ miteinander vergleichen zu können, wurden subjektive und objektive Messwerte erhoben. Die objektiven Fahrdaten aus dem Simulator wurden aufgezeichnet.

Da zur Rückübernahmefähigkeit bei unterschiedlich beanspruchenden FFT im Stadtverkehr bisher keine Erkenntnisse vorliegen, wurde diese in Studie 2 untersucht. Bei dem Versuch wurde ein Within-Subject-Design angewendet. Hierbei führen alle Proband_innen die FFT in mehreren Durchgängen aus. Während jeder zu untersuchenden FFT gab es eine Rückübernahmeaufforderung. Aufgrund der Ergebnisse aus Studie 1 wurde nur der multimodale TOR verwendet. Die Probanden_innen wurden angewiesen, dass sie ihre Priorisierung auf die FFT und nicht auf den Straßenverkehr lenken sollen. Bei allen Rückübernahmeszenarien wurde auf zusätzlichen Verkehr verzichtet, um den Einflussfaktor der Verkehrsdichte konstant zu halten (Radlmayr et al. 2014). Der TOR findet jeweils auf gerader Strecke bei einer Geschwindigkeit von $50 \mathrm{~km} / \mathrm{h}$ nach Überfahren eines definierten Wegpunktes statt. Bei der Rückübernahme muss die Versuchsperson eine drohende Kollision durch ein Ausweichen oder Abbremsen verhindern. Der schematische Ablauf eines TOR ist in Abb. 3 dargestellt.

Die Reaktionszeit auf einen TOR entspricht der Zeitspanne zwischen dem Start der TOR-Ausgabe und der Reaktion des Fahrers/der Fahrerin. Als Reaktion wird die Betätigung von Lenkrad und/oder Pedal gezählt. Eingriffe durch das Lenkrad werden ab einer Winkeländerung von $2^{\circ}$ und Eingriffe durch die Betätigung des Gas- oder Bremspedals bei Änderungen der Pedalstellung um mehr als $10 \%$ gegenüber der Ausgangsposition berücksichtigt. Nach jedem TOR wurde mit einem Fragebogen die wahrgenommene Dringlichkeit, Benutzerfreundlichkeit, Ablenkung und den durch den TOR vermittelten Komfort auf einer 7-Punkte-Likert-Skala durch die Proband_innen TOR bewertet.
Vor Beginn der Versuchsfahrt wurde den Proband_innen die Funktionsweise eines automatisierten Fahrzeuges erklärt. Dabei erhielten die Probanden auch die Information, dass eine Fokussierung der eigenen Aufmerksamkeit auf die Fahrsituation nicht mehr notwendig ist und FFT während der Fahrt ausgeführt werden können.

\subsection{Datenauswertung}

Zur Darstellung der untersuchten Parameter wurden Boxplot-Diagramme verwendet und zusätzlich das arithmetische Mittel angegeben. Die statistische Auswertung erfolgte auf dem Signifikanzniveau von $\alpha=0,05$. Für die Prüfung auf Normalverteilung wurde der Shapiro-Wilk-Test verwendet. Sofern eine Normalverteilung der beiden Stichproben vorlag, wurde in Studie $1 c$ ein t-Test für abhängige Stichproben durchgeführt. Zum Vergleich von Studie $1 b$ und Studie $1 c$ wurde ein $\mathrm{t}$-Test für unabhängige Stichproben verwendet. Dabei wurde die Homogenität der Varianz mit dem Levene-Test überprüft. Als Ergebnis des Mittelwertvergleichs kann eine Vorhersage getroffen werden, ob sich die betrachteten Mittelwerte signifikant voneinander unterscheiden $(p \leq 0,05)$ oder nicht.

\section{Ergebnisse}

\subsection{Bewertung der Vibrationsintensitäten}

In Studie $1 a$ wurden unterschiedliche Vibrationsintensitäten zur Auslegung der Vibrationsmatte von den Proband_innen bewertet. Die Ergebnisse zeigen, dass niedrige Vibrationsstärken $(0,7 \mathrm{~V} / 30 \mathrm{~Hz} / 0,3 \mathrm{~g})$ als angenehm und hohe Vibrationsstärken $(3 \mathrm{~V} / 105 \mathrm{~Hz} / 6 \mathrm{~g})$ als eher unangenehm empfunden werden (Abb. 4). Wie auch in Ji et al. (2011) beschrieben, wurde eine Geschlechtsabhängigkeit bei der Vibrationswahrnehmung festgestellt. Frauen bewerteten die Vibrationsintensität im Bereich von 1,2 bis 2,0 V als unangenehmer im Vergleich zu Männern. Bei niedrigen und hohen
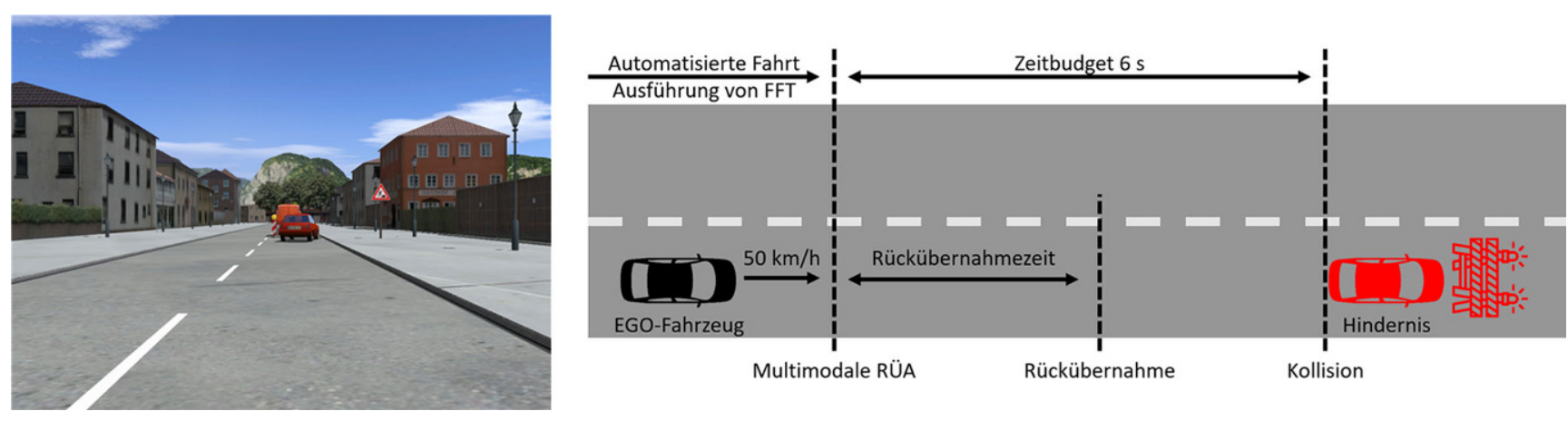

Abb. 3 Ablauf eines TOR in Studie 2

Fig. 3 Procedure of a TOR in study 2 


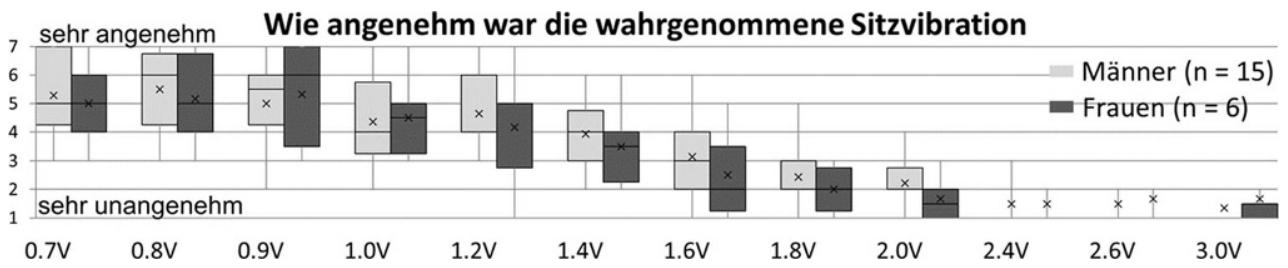

Abb. 4 Subjektive Bewertung der wahrgenommenen Vibration in Abhängigkeit von Geschlecht und Vibrationsstärke Fig. 4 Subjective evaluation of the perceived vibration as a function of gender and vibration intensity

Abb. 5 Reaktionszeiten [s] zwischen dem TOR und dem Eingreifen des Fahrers/der Fahrerin Fig. 5 Reaction times [s] between the TOR and the intervention of the driver

\section{Reaktionszeiten in sek zwischen dem TOR und dem Eingreifen des Fahrzeugnutzenden}

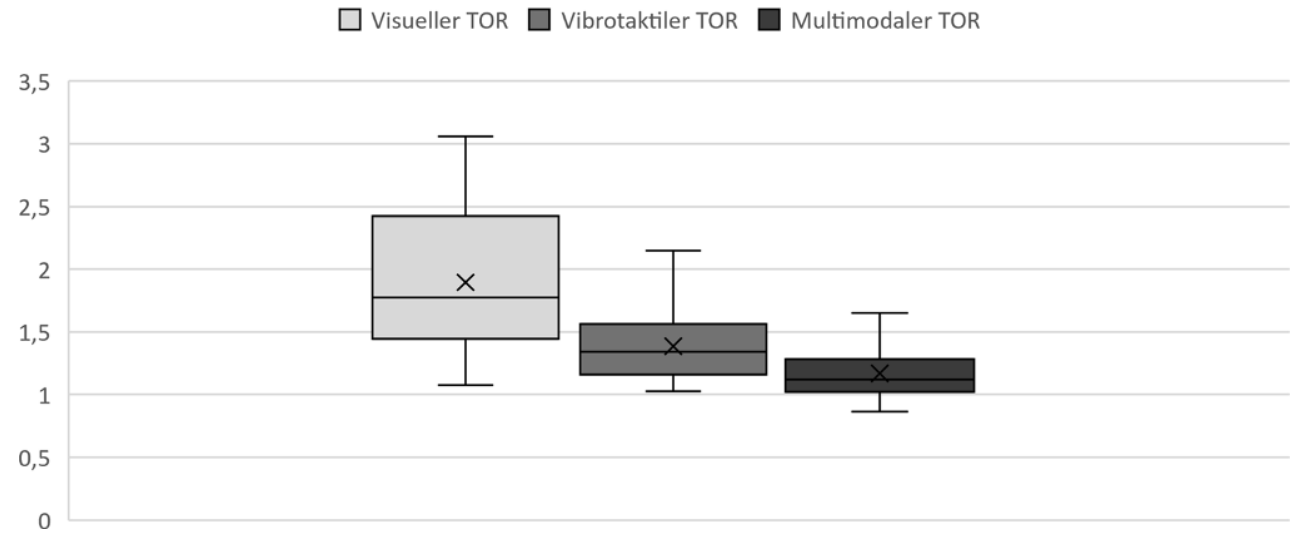

Vibrationsintensitäten gibt es nur geringe Geschlechtsunterschiede. Aufgrund der geringen Anzahl von Proband_innen wurde auf eine Inferenzstatistik verzichtet. Aufgrund der Ergebnisse der Studie $1 a$ wurde als optimale Vibrationsstärke ein Wert von 1,4 V gewählt, da dieser von den Proband_innen als Mittelwert zwischen sehr angenehm und sehr unangenehm bewertet wurde. Es ist zu erwarten, dass die Proband_innen in den weiteren Studien weder die Vibration aufgrund einer zu geringen Intensität nicht wahrnehmen noch durch eine zu hoch eingestellte Vibrationsintensität zu sehr abgelenkt werden.

\subsection{Reaktionszeiten nach einem TOR}

Im Folgenden werden die Reaktionszeiten aus Studie $1 b$ und Studie $1 c$ zwischen dem initiierten TOR und dem Eingriff des Fahrers/der Fahrerin zusammengefasst und erläutert.

Tab. 2 Mittelwerte und Standardabweichungen für die Reaktionszeiten zwischen dem TOR und dem Eingreifen des Fahrers/der Fahrerin

Table 2 Mean values and standard deviations for the reaction times between the TOR and the intervention of the driver

\begin{tabular}{lll}
\hline TOR-Variante & Mittelwert [s] & Standardabweichung [s] \\
\hline Visuell & 1,89 & 0,60 \\
Vibrotaktil & 1,39 & 0,27 \\
Multimodal & 1,17 & 0,20 \\
\hline
\end{tabular}

In allen drei Szenarien treten die längsten Reaktionszeiten bei dem visuellen TOR und die kürzesten bei dem multimodalen TOR auf, die Mittelwertsunterschiede sind zum Teil signifikant (siehe Abb. 5).

Für die über alle drei Szenarien gemittelten Reaktionszeiten, ergeben sich die in Tab. 2 dargestellten Mittelwerte und Standardabweichungen.

Die mittleren Reaktionszeiten bei dem multimodalen TOR sind signifikant kürzer als bei dem visuellen TOR $(\mathrm{t}(20,835)=5,055, p=0,000, \mathrm{~d}=0,74)$. Ebenso sind die $\mathrm{Re}$ aktionszeiten bei dem multimodalen TOR signifikant kürzer als bei dem vibrotaktilen TOR $(\mathrm{t}(26)=5,215, p=0,000$, $\mathrm{d}=0,72)$.

\subsection{Subjektive Bewertungen der TOR-Varianten}

Zusätzlich zu den objektiven Fahrdaten wurde den Proband_innen nach jeder TOR ein Fragebogen in Studie $1 b$ und $1 c$ ausgehändigt. Die Ergebnisse der subjektiven Befragung sind in Abb. 6 dargestellt.

Hinsichtlich der empfundenen Dringlichkeit werden der visuelle $(\mathrm{t}(47)=2,44, p=0,019, \mathrm{~d}=0,34)$ sowie der multimodale TOR $(\mathrm{t}(29)=-4,05, p=0,000, \mathrm{~d}=0,60)$ signifikant besser bewertet als die vibrotaktile Variante.

Der visuelle TOR wird tendenziell als hilfreicher empfunden als der vibrotaktile TOR. Als signifikant hilfreicher wird der multimodale TOR im Vergleich zum vibrotaktilen TOR bewertet $(\mathrm{t}(29)=-2,446, p=0,021, \mathrm{~d}=0,41)$. 
Abb. 6 Subjektive Bewertungen der untersuchten TOR-Varianten

Fig. 6 Subjective ratings of examined TOR variants

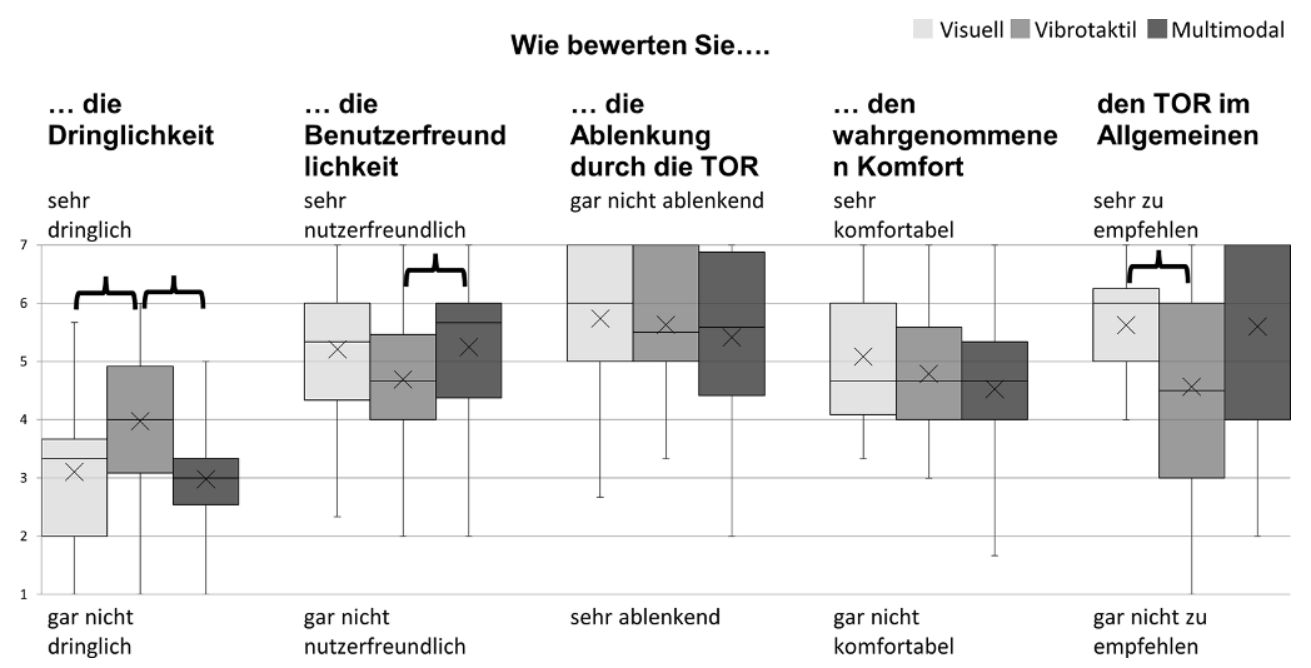

Es zeigt sich, dass die Mehrheit der Proband_innen das Warnsystem nicht als störend empfindet. Zwischen den drei TOR Varianten können keine signifikanten Unterschiede festgestellt werden.

Auch hinsichtlich des empfundenen Komforts bestehen keine signifikanten Unterschiede zwischen den verschiedenen Varianten.

Schließlich wurden die drei TOR-Varianten zusammenfassend auf einer 7-Punkte-Likert-Skala bewertet. Der visuelle TOR wird signifikant besser bewertet als der vibrotaktile TOR $(\mathrm{t}(43,716)=2,396, p=0,021, \mathrm{~d}=0,34)$. In der durchschnittlichen Bewertung unterscheiden sich der visuelle und der multimodale TOR nicht voneinander.

\subsection{Reaktionszeit in Abhängigkeit von verschiedenen $\mathrm{FFT}$}

In Studie 2 wurden Reaktionszeiten auf einen multimodalen TOR bei den FFT Text lesen, Hörbuch hören, Video schauen und Texting während der automatisierten Fahrt untersucht. Zusätzlich wurden die Reaktionszeiten auf einen TOR bei der Referenztätigkeit Straße beobachten analysiert.

Die längste durchschnittliche Reaktionszeit wurde bei der FFT Text lesen gemessen $(M=1,64 \mathrm{~s}, \mathrm{SD}=0,31 \mathrm{~s}$,
90. Perzentil $=2,48 \mathrm{~s}$ ). Diese ist signifikant länger als bei allen anderen untersuchten FFT. Die FFT Film schauen $(\mathrm{M}=1,48 \mathrm{~s}, \mathrm{SD}=0,24 \mathrm{~s}, 90$. Perzentil=2,49s $)$ und Texting $(M=1,49 s, S D=0,28 s, 90$. Perzentil=3,42s) liegen in einem sehr ähnlichen Bereich und die Übernahmezeit ist signifikant länger als beim Hörbuch hören $(\mathrm{M}=1,10 \mathrm{~s}$, $\mathrm{SD}=0,25 \mathrm{~s}$, 90. Perzentil $=1,83 \mathrm{~s}$ ) und Straße beobachten $(\mathrm{M}=1,11 \mathrm{~s}, \mathrm{SD}=0,38 \mathrm{~s}, 90$. Perzentil=3,02s). Die beiden letztgenannten unterscheiden sich nicht voneinander. Eine angepasste Varianzanalyse zeigt einen starken Effekt, (Greenhouse Geisser $\mathrm{F}(3,32,172,86)=54,46, p<0,001$, $\mathrm{f}=0,59)$. Die Ergebnisse sind in Abb. 7 und Tab. 3 dargestellt.

In einer detaillierteren Analyse wurde der Zusammenhang zwischen der Reaktionszeit und dem Konstrukt mentale Beanspruchung ermittelt. Die mentale Beanspruchung wurde für jede FFT mit der Methode Detection Response Task gemessen. FFT mit einer hohen mentalen Beanspruchung führen zu längeren Reaktionszeiten. Eine Regressionsanalyse zeigt, dass die Übernahmezeit mit zunehmender mentaler Beanspruchung signifikant zunimmt $\left(\mathrm{F}(1,268)=30,74, p<0,001, \mathrm{R}^{2}=0,103\right)$.
Abb. 7 Boxplot-Darstellung der Reaktionszeit [s] in Abhängigkeit von der untersuchten FFT

Fig. 7 Boxplot representation of the reaction time $[\mathrm{s}]$ depending on the examined non-driving related task (NDRT) DRT in $\mathrm{s}$

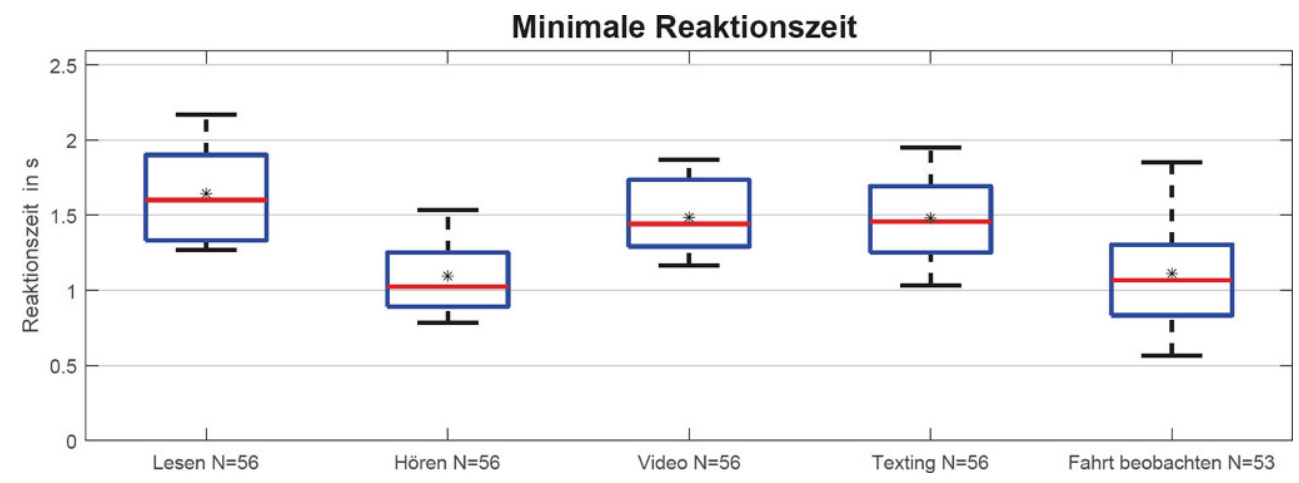


Tab. 3 Überblick über die Ergebnisse zu den Reaktionszeiten $[\mathrm{s}]$

Table 3 Overview of the results on reaction times $[\mathrm{s}]$

\begin{tabular}{lllllll}
\hline FFT & MW & SD & & Mittelwertdifferenz [s] & \\
\cline { 5 - 7 } & & & Lesen & $\begin{array}{l}\text { Hörbuch } \\
\text { hören }\end{array}$ & $\begin{array}{l}\text { Film } \\
\text { schauen }\end{array}$ & Texting \\
\hline Lesen & 1,64 & 0,31 & - & - & - & - \\
Hörbuch hören & 1,10 & 0,25 & $\mathbf{0 , 5 4 *}$ & - & - & - \\
Film schauen & 1,48 & 0,24 & $\mathbf{0 , 1 6 *}$ & $\mathbf{- 0 , 3 8 *}$ & - & - \\
Texting & 1,49 & 0,28 & $\mathbf{0 , 1 5 *}$ & $\mathbf{- 0 , 3 9 *}$ & $-0,01$ & - \\
Fahrt beobachten & 1,11 & 0,38 & $\mathbf{0 , 5 3 *}$ & $-0,00$ & $\mathbf{0 , 3 7}^{*}$ & $\mathbf{0 , 3 8 *}$ \\
\hline
\end{tabular}

*Signifikanter Unterschied in der Mittelwertdifferenz ( $\alpha=0,05$; Bonferroni-korrigiert)

\section{Diskussion}

Die Diskussion geht zunächst auf die Gestaltung der TORVarianten ein. Anschließend werden die TOR-Varianten hinsichtlich der Reaktionszeiten und subjektiven Bewertungen verglichen. Abschließend werden die Forschungsfragen beantwortet.

\subsection{TOR-Gestaltung}

Die Ergebnisse haben gezeigt, dass der visuelle TOR aufgrund seiner alarmierenden roten Pulsfrequenz intuitiv verständlich ist und sich gut als Warnsystem eignet. Trotz der Durchführung einer FFT und der damit verbundenen Blickabwendung von der Straße ist die visuellen Rückübernahmeaufforderung im peripheren Sichtfeld des Fahrers noch gut sichtbar. Proband_innen aus Studie $1 b$ sagten, dass eine akustische Warnung zusätzlich zum visuellen Stimulus unterstützen würde. Prinzipiell empfanden die Probanden das System nicht als störend. Lediglich Reflexionseffekte auf der Windschutzscheibe des Fahrzeug-Mock-up wurden negativ wahrgenommen.

Das akustische TOR-Signal wurde von den Probanden in Studie $1 c$ positiv beurteilt. Der Warnton von $440 \mathrm{~Hz}$ wurde trotz simulierter Verkehrs- und Windgeräusche sowie der durchgeführten FFT von allen Teilnehmenden wahrgenommen. In der durchgeführten Studie wurde das akustische Signal nur in Kombination mit dem vibrotaktilen und dem visuellen TOR getestet.

Für den vibrotaktilen TOR wurde auf Basis der Ergebnisse aus Studie $1 a$ für die verwendeten Aktuatoren (Precision Dynamics 320-105) eine Schwingungsfrequenz von ca. $50 \mathrm{~Hz}$ und eine Schwingungsamplitude von $1,5 \mathrm{~g}$ bei einer angelegten Spannung von 1,4 V angewendet. Es ist davon auszugehen, dass die Fahrer_innen weder die Vibration aufgrund einer zu geringen Vibrationsintensität nicht wahrnehmen, noch durch eine zu hohe Vibrationsintensität erschreckt werden, was zu einer schlechteren Übernahmequalität führen würde. Die subjektiven Bewertungen der Vibrationsintensität haben geschlechtsspezifische Unterschiede gezeigt. Frauen bewerteten die empfundene Vibrationsintensität insbesondere im mittleren Spannungs- bereich (1,2-2,0 V) als unangenehmer als Männer (vgl. Ji et al. 2011). Ob dieser Effekt tatsächlich vom Geschlecht oder vom Körpergewicht abhängt, sollte jedoch in weiteren Studien untersucht werden. Einzelne Proband_innen empfanden die Einwirkung der Vibrationen in verschiedenen Körperbereichen als sehr unangenehm. Die Angaben variieren zwischen den Proband_innen, so dass keine allgemein gültige Aussage getroffen werden kann. Dennoch werden der Rückenbereich im Allgemeinen und der Nierenbereich im Besonderen häufiger genannt.

\subsection{Reaktionszeiten}

Eines der wichtigsten Kriterien für die Bewertung eines TOR sind kurze Reaktionszeiten, um kritische Situationen zu vermeiden. Die hier durchgeführten Versuche zeigen, dass die kürzesten Reaktionszeiten mit einem multimodalen TOR erreicht werden, gefolgt vom vibrotaktilen und dem visuellen TOR.

Ein möglicher Grund, warum der visuelle TOR zu längeren Reaktionszeiten führte, könnte darin liegen, dass die Proband_innen zum Zeitpunkt des TOR eine visuelle FFT ausgeführt haben. Dieser visuelle Stimulus während eines visuellen TOR könnte zu einer Verzögerung der Informationsverarbeitung und der Handlungsausführung führen. Dies spricht auch für die multiple Ressourcentheorie nach Wickens (2008). Ob ähnliche Effekte auftreten, wenn eine akustische FFT bei einem akustischen TOR durchgeführt wird, sollte weiter untersucht werden.

\subsection{Subjektive Bewertung}

Die subjektiven Bewertungen der TOR-Varianten untermauern die Ergebnisse hinsichtlich der festgestellten Reaktionszeiten. Die Proband_innen äußerten, dass der visuelle TOR effektiv über die Notwendigkeit des Eingreifens informiere, da eine direkte Handlungsaufforderung in Bezug auf den relevanten Bereich, in diesem Fall die Windschutzscheibe und damit das äußere Verkehrsgeschehen, gegeben sei. Bei dem vibrotaktilen TOR ist der Vibrationsreiz nicht mit einer Handlungsaufforderung verbunden und die Proband_innen wussten oft nicht genau, was zu tun ist. Die 
Bewertung der wahrgenommenen Benutzerfreundlichkeit bestätigt, dass ein vibrotaktiles Signal allein nicht hilfreich ist. Die Ablenkungswirkung unterscheidet sich nicht zwischen den drei TOR-Varianten und wird von der Mehrheit der Probanden nicht als störend empfunden.

\subsection{Reaktionszeiten bei unterschiedlich beanspruchenden FFT}

Eine wesentliche Frage bei der Untersuchung von hochautomatisierten Fahrsystemen ist, ob der Mensch im Falle eines TOR schnell wieder die Kontrolle über das Fahrzeug übernehmen kann.

Wie oben in Abschn. 2 Teil beschrieben, hängt die Übernahmezeit von verschiedenen Faktoren ab. Der Einfluss der Gestaltung des TOR wurde im Vorfeld in den Studien $1 a$ bis $1 c$ empirisch untersucht und die beste Variante ausgewählt. Insgesamt erlebten die Versuchsteilnehmenden während der gesamten Studie 2 sechs TOR-Situationen (Eine bei der Eingewöhnungsfahrt und jeweils eine pro FFT). Es konnten keine Lerneffekte in Abhängigkeit von der Anzahl der erlebten TOR festgestellt werden. Signifikante Unterschiede wurden bei der Rückübernahmezeit in Abhängigkeit von den untersuchten FFT festgestellt. Die Mittelwerte der Übernahmezeit liegen in dieser Studie zwischen $1,10 \mathrm{~s}$ (Hören) und 1,64 s (Lesen) und damit in einem kürzeren Bereich als in der vorliegenden Literatur. Ein Grund dafür könnte der hier eingesetzte multimodale TOR sein.

Zudem unterscheiden sich die Ergebnisse dieser Arbeit von Studien, die ebenfalls verschiedene FFT untersucht haben. Von Vogelpohl et al. (2016) und von Zeeb et al. (2016) wurden keine Unterschiede zwischen den Übernahmezeiten bei unterschiedlichen FFT gefunden. Eine mögliche Erklärung hierfür könnte darin liegen, dass die FFT in Studie 2 explizit priorisiert werden sollte und die Teilnehmenden auch zum Inhalt der FFT befragt wurden. Was im Umkehrschluss bedeutet, dass die Versuchsteilnehmenden noch stärker in die FFT eingebunden waren.

\subsection{Zusammenfassende Beantwortung der Forschungsfragen}

Forschungsfrage 1 Ist ein unimodaler TOR ausreichend, um eine schnelle Reaktion des Fahrers/der Fahrerin zu erzielen oder führt eine gleichzeitige multimodale Ansprache verschiedener Sinneskanäle zu besseren Reaktionszeiten?

Die Ergebnisse zeigen, dass alle drei TOR-Varianten ihren Zweck erfüllen und alle Proband_innen vom automatisierten Fahrmodus zurück zum manuellen Fahren wechselten, indem sie wieder das Lenkrad oder die Pedale benutzten.

Basierend auf den hier durchgeführten Untersuchungen sollte ein multimodaler TOR bevorzugt werden, da mit die- sem die kürzesten Reaktionszeiten in kritischen und unkritischen Verkehrssituationen sowie durchweg gute subjektive Bewertungen erreicht wurden. Zukünftige Studien sollten die Parametrisierung einzelner Faktoren der multimodalen TOR fortsetzen und mit einem einheitlichen Testdesign optimieren.

Die gemessenen Reaktionszeiten weisen darauf hin, dass in der untersuchten Fragestellung die Verwendung multimodaler Stimuli die beste Reiz-Reaktions-Kompatibilität zeigen. Dies ist auch damit zu erklären, dass in Abhängigkeit der Art der FFT unterschiedliche Ressourcen des Menschen beansprucht werden, so dass eine Informationsdarbietung über unterschiedliche Modalitäten die Nutzung freier Ressourcen fördert.

Forschungsfrage 2 Wie unterscheidet sich die Rückübernahmefähigkeit bei der Ausführung verschiedener FFT?

Bei den Rückübernahmezeiten in Abhängigkeit von den untersuchten FFT wurden signifikante Unterschiede festgestellt. Die Mittelwerte der Übernahmezeit liegen in dieser Studie zwischen 1,10s (Hören) und 1,64s (Lesen). Aufgrund der durch die unterschiedlichen FFT vorwiegend beanspruchten Sinneskanäle hat es sich als vorteilhaft erwiesen, einen multimodalen TOR einzusetzen und somit auch die durch die FFT weniger stark beanspruchten Wahrnehmungskanäle zu adressieren.

Danksagung Ein Teil der vorliegenden Arbeit ist ein Ergebnis des Verbundforschungsprojektes @CITY - Automated Cars and Intelligent Traffic in the City. Das Forschungsvorhaben wird vom Bundesministerium für Wirtschaft und Energie (BMWi) aufgrund eines Beschlusses des Deutschen Bundestages gefördert. Die Verantwortung für den Inhalt dieser Veröffentlichung tragen allein die Verfasser.

Funding Open Access funding enabled and organized by Projekt DEAL.

Open Access Dieser Artikel wird unter der Creative Commons Namensnennung 4.0 International Lizenz veröffentlicht, welche die Nutzung, Vervielfältigung, Bearbeitung, Verbreitung und Wiedergabe in jeglichem Medium und Format erlaubt, sofern Sie den/die ursprünglichen Autor(en) und die Quelle ordnungsgemäß nennen, einen Link zur Creative Commons Lizenz beifügen und angeben, ob Änderungen vorgenommen wurden.

Die in diesem Artikel enthaltenen Bilder und sonstiges Drittmaterial unterliegen ebenfalls der genannten Creative Commons Lizenz, sofern sich aus der Abbildungslegende nichts anderes ergibt. Sofern das betreffende Material nicht unter der genannten Creative Commons Lizenz steht und die betreffende Handlung nicht nach gesetzlichen Vorschriften erlaubt ist, ist für die oben aufgeführten Weiterverwendungen des Materials die Einwilligung des jeweiligen Rechteinhabers einzuholen.

Weitere Details zur Lizenz entnehmen Sie bitte der Lizenzinformation auf http://creativecommons.org/licenses/by/4.0/deed.de. 


\section{Literatur}

Carsten O, Lai FCH, Barnard Y, Jamson AH, Merat N (2012) Control task substitution in semiautomated driving. Does it matter what aspects are automated? Hum Factors 54(5):747-761

Damböck D, Farid M, Tönert L, Bengler K (2012) Übernahmezeiten beim hochautomatisierten Fahren. Tagung Fahrerassistenz, München

DIN ISO/TS 14198 (2019) Straßenfahrzeuge - Ergonomische Aspekte von Fahrerinformations- und Assistenzsystemen - Kalibrierungsaufgaben für Methoden, welche auf Fahreranfragen zugreifen, um fahrzeuginterne Systeme zu verwenden. Beuth, Berlin

Donges E (1982) Aspekte der aktiven Sicherheit bei der Führung von Personenkraftwagen. AUTOMOB IND 27:2

Eriksson A, Marcos IS, Kircher K, Västfjäll D, Stanton NA (2015) The development of a method to assess the effects of traffic situation and time pressure on driver information preferences. In: Harris D (Hrsg) Engineering psychology and cognitive ergonomics. Lecture notes in computer science. Springer, Cham, S 3-12 https:// doi.org/10.1007/978-3-319-20373-7_1

Färber B (1987) Geteilte Aufmerksamkeit. Grundlagen und Anwendung im motorisierten Strassenverkehr. Mensch, Fahrzeug, Umwelt Bd. 20. TÜV Rheinland, Köln

Gold C, Körber M, Lechner D, Bengler K (2016) Taking over control from highly automated vehicles in complex traffic situations: the role of traffic density. Hum Factors 58(4):642-652

Happee R, Gold C, Radlmayr J, Hergeth S, Bengler K (2017) Take-over performance in evasive manoeuvres. Accid Anal Prev 106:211-222

Ji YG, Lee K, Hwang W (2011) Haptic perceptions in the vehicle seat. Hum Factors Man 21(3):305-325

Kirchner WK (1958) Age differences in short-term retention of rapidly changing information. J Exp Psychol 55(4):352-358

Louw T, Merat N, Jamson H (2015) Engaging with highly automated driving: to be or not to be in the loop? In: Holeton KM (Hrsg) Driving assessment conference. 8th international driving symposium on human factors in driver assessment. Training and vehicle design. University of Iowa, Iowa City

Merat N, Jamson H, Lai F, Carsten O (2012) Highly automated driving, secondary task performance, and driver state. Hum Factors 54(5):762-771

National Highway Traffic Safety Administration (2012) Visual-manual NHTSA driver distraction guidelines for in-vehicle electronic devices NHTSA-2010-0053

Naujoks F, Mai C, Neukum A (2014) The effect of urgency of take-over requests during highly automated driving under distraction conditions. In: Jang R-L, Ahram T (Hrsg) Advances in human factors and ergonomics 2014 5th International Conference on Applied
Human Factors and Ergonomics : 20 volume set: proceedings of the 5th AHFE Conference, 19-23 July 2014

Petermann-Stock I, Hackenberg L, Muhr T, Mergl C (2013) Wie lange braucht der Fahrer? Eine Analyse zu Übernahmezeiten aus verschiedenen Nebentätigkeiten ährend einer hochautomatisierten Staufahrt. In: TÜV SÜD (Hrsg) Der Weg zum automatischen Fahren. 6. Tagung Fahrerassistenz, S 1-26

Petermeijer S, Bazilinskyy P, Bengler K, de Winter J (2017a) Takeover again. Investigating multimodal and directional TORs to get the driver back into the loop. Appl Ergon 62:204-215

Petermeijer SM, Cieler S, de Winter JCF (2017b) Comparing spatially static and dynamic vibrotactile take-over requests in the driver seat. Accid Anal Prev 99(Pt A):218-227

Radlmayr J, Gold C, Lorenz L, Farid M, Bengler K (2014) How traffic situations and non-driving related tasks affect the take-over quality in highly automated driving. Proc Hum Factors Ergon Soc Annu Meet 58(1):2063-2067

SAE J3016 (2021) Taxonomy and definitions for terms related to driving automation systems for on-road motor vehicles. Society of Automotive Engineers. https://www.sae.org/standards/content/ j3016_202104/. Zugegriffen: 28. Sept. 2021

Strand N, Nilsson J, Karlsson IM, Nilsson L (2014) Semi-automated versus highly automated driving in critical situations caused by automation failures. Transp Res Part F Traffic Psychol Behav 27:218-228

Strasser H (2008) Kompatibilität, Leitlinie für ergonomische Arbeitsgestaltung und präventiven Arbeitsschutz. In: Produkt- und Produktions-Ergonomie - Aufgabe für Entwickler und Planer Proceedings des 54. Arbeitswissenschaftlichen Kongresses der Gesellschaft für Arbeitswissenschaft. GfA Press, Dortmund, S $17-28$

Telpaz A, Rhindress B, Zelman I, Tsimhoni O (2017) Using a vibrotactile seat for facilitating the handover of control during automated driving. Int J Mob Hum Comput Interact 9(3):17-33

Utesch F (2014) Unscharfe Warnungen im Kraftfahrzeug. Eignet sich eine LED-Leiste als Anzeige für Fahrerassistenzsysteme? Dissertation. Technische Universität Carolo-Wilhelmina, Braunschweig

Vogelpohl T, Vollrath M, Kühn M, Hummel T, Gehlert T (2016) Übergabe von hochautomatisiertem Fahren zu manueller Steuerung. Forschungsbericht Nr. 39. Gesamtverband der Deutschen Versicherungswirtschaft e. V, Berlin

Wickens CD (2008) Multiple resources and mental workload. Hum Factors 50(3):449-455

Zeeb K, Buchner A, Schrauf M (2016) Is take-over time all that matters? The impact of visualcognitive load on driver take-over quality after conditionally automated driving. Accid Analysis Prev 92:230-239 